Communication

\title{
Adipose Tissue as a Useful Material for the Grafting of Tumorigenic Cells and Juvenile Tissues in Mice
}

Masahiro Sato ${ }^{1, \neq,^{*}}$, Yuki Kiyokawa ${ }^{2}$, Emi Inada $^{3}$, Eri Akasaka ${ }^{1}$, Satoshi Watanabe ${ }^{4}$, Issei Saitoh ${ }^{2}$

1. Section of Gene Expression Regulation, Frontier Science Research Center, Kagoshima University, Kagoshima 890-8544, Japan; E-Mails: sato-masa@ncchd.go.jp; stylistics777@yahoo.co.jp

2. Department of Pediatric Dentistry, Asahi University School of Dentistry, Gifu 501-0296, Japan; EMails: ykiyokawa@dent.asahi-u.ac.jp; isaitoh@dent.asahi-u.ac.jp

3. Department of Pediatric Dentistry, Graduate School of Medical and Dental Sciences, Kagoshima University, 890-8544 Kagoshima, Japan; E-Mail: inada@dent.kagoshima-u.ac.jp

4. Animal Genome Unit, Institute of Livestock and Grassland Science, National Agriculture and Food Research Organization (NARO), 2 Ikenodai, Tsukuba, Ibaraki 305-0901, Japan; E-Mail: kettle@affrc.go.jp

¥ Current Affiliation: Department of Genome Medicine, National Center for Child Health and Development, 2-10-1 Okura, Setagaya, Tokyo 157-8535, Japan

* Correspondence: Masahiro Sato; E-Mail: sato-masa@ncchd.go.jp

Academic Editor: Haval Shirwan

Special Issue: Abdominal Organ Transplantation

OBM Transplantation

2021, volume 5 , issue 4

doi:10.21926/obm.transplant. 2104155
Received: March 15, 2021

Accepted: October 19, 2021

Published: November 04, 2021

\begin{abstract}
Although the aging process expands the adipose tissue habitation in mice and due to its close association with the female reproductive system, it can be easily exposed surgically under anesthesia when reproductive organs (including ovary, oviduct, and part of the uterus) are pulled and exposed onto the dorsal skin. This study aimed to consider the suitability of adipose tissue as a target for manipulation, particularly for the grafting of cells or small-sized tissue sections due to its ease of handling. Subsequently, 1-2 $\mu \mathrm{L}$ trypan blue injections were
\end{abstract}

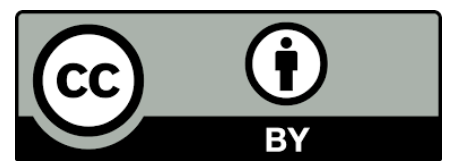

(C) 2021 by the author. This is an open access article distributed under the conditions of the Creative Commons by Attribution License, which permits unrestricted use, distribution, and reproduction in any medium or format, provided the original work is correctly cited. 
administered to the tissues using a breath-controlled micropipette under a dissecting microscope for evaluating the adipose tissue's potential as a suitable grafting material. It was observed that the injected dye remained at the injection site for at least one day after injecting B16 mouse melanoma and P19 embryonal carcinoma cells. It resulted in the generation of solid tumors surrounding the ovary, oviduct, or uterus with $100 \%$ efficiency, as reported by an inspection one and a half months after the injections. When the grafting procedure was carried out for one-fourth of the juvenile pancreas (aged 15 days), an enlarged pancreas with normal morphological configuration (including the formation of insulin-synthesizing cells) was generated, which was observed by an inspection one month after the injections, thus successfully validating our approach for adipose tissue manipulation and furthermore, naming this novel technology as "intra-adipose introduction of cells and tissues".

\section{Keywords}

Adipose tissue; transplantation; solid tumor; juvenile pancreas; B16 melanoma; P19 embryonal carcinoma; In vivo teratoma formation assay

\section{Introduction}

Recent advancements in pluripotent stem cell research have widened the possibility of several regenerative strategies for patient-specific cell therapy, novel drug therapy as well as disease remodeling. Induced pluripotent stem cells (iPSCs)/embryonic stem cells (ESCs) have the innate potential to differentiate into fully differentiated cells originating from the three germ layers, when precisely cultured under differentiation-inducing conditions [1, 2]. Since both in vitro and in vivo approaches are available for assessing the differentiation potential of these cells, the former component includes cultivation of iPSCS/ESCs in a medium supplemented with differentiationinducing reagents, whereas the latter aspect involves the inoculation of immunocompromised mice, such as nude and non-obese diabetic/severe combined immunodeficient (NOD/SCID) mice, at growth-permissive sites. The in vivo approach also known as the "in vivo teratoma formation assay" involves the formation of solid tumors named "teratoma", by the inoculated cells containing various types of differentiated cells originating from the three germ layers [1-9].

Several suitable sites for iPSCs/ESCs transplantation involving the in vivo grafting of tumorigenic mice cells (particularly in immunocompromised mice, such as nude mice or NOD/SCID mice) are available out of which the most common locations are subcutaneous cell grafting and cell grafting under the renal capsule [10-17] followed by intratesticular grafting [13, 18-22], intramuscular grafting [23-28], intramyocardial grafting [23], cochlear grafting [29], liver parenchyma [10], and salivary glands grafting [30]; however, a large number of tumor cells are required for inoculation to achieve successful tumorigenesis [31]. Notably, Prokhorova et al. [11] reported that co-injecting human ESCs with Matrigel increased subcutaneous teratoma formation efficiency from $25-40 \%$ to 80-100\% while suggesting that as Matrigel, a gelatinous extracellular matrix was produced from Engelbreth-Holm-Swarm (EHS) mouse sarcoma cells, it enhanced survival and growth of teratoma and other tumors through interaction with several matrix-associated growth factors and limited the implanted cells migration away from the site of implantation, thereby potentially enhancing their 
paracrine interactions while assuming that the spreading of inoculated cells from the implantation site was indeed a critical factor determining the success of the in vivo teratoma formation assay.

Henceforth, a novel method named "intrapancreatic parenchymal cell transplantation" (IPPCT) was developed, based on the transplantation of actively proliferating tumor cells (including iPSCs) into the nude mice pancreatic parenchyma using a mouthpiece-controlled glass micropipette under observation with a dissecting microscope for minimizing the spread of inoculated cells and increasing the in vivo teratoma formation efficiency [32], and it was observed that the cells or cellular aggregates inoculated within the pancreatic compartment did not spread easily beyond the compartment while a success rate of almost $100 \%$ was achieved in the formation of solid tumors (including teratoma) using IPPCT. Since the pancreas can be exposed easily by removing the spleen after a skin incision, IPPCT appears to be an easy and useful technology for inducing the in vivo growth of cells into solid tumors.

Owing to the fact that adipose tissue is a loose connective tissue composed mostly of adipocytes [33], and can be easily exposed during surgeries involving the removal of female reproductive system organs (i.e., ovary, oviduct, or uterus) by the skin incisions, due to proximity with female reproductive organs. Therefore, the ease of handling organs such as the pancreas indicates that adipose tissue can be easily used for inducing the growth of tumorigenic cells as solid tumors.

This study involved the transplantation of actively proliferating tumor cells [including B16 melanoma cells and P19 embryonal carcinoma cells (ECCs)] into mice adipose tissue using the IPPCT technique to determine whether these cells could grow as solid tumors in vivo. Furthermore, a small piece of the juvenile pancreas was also transplanted into the adipose tissue to observe whether the grafted tissues could grow and function as insulin-producing tissues, and this novel procedure was named "intra-adipose introduction of cells and tissues" (IAdICT).

\section{Materials and Methods}

\subsection{Animals}

For the tumor cells inoculation into the adipose tissue, 8-20-week-old immunodeficient female mice (BALB/c-nu/nu; CLEA Japan Ltd., Tokyo, Japan) were used while some cases involved the usage of adult (four to ten months old) or juvenile (15 days old) female B6C3F1 mice (a hybrid between C57BL/6 and C3H/He; CLEA Japan Ltd.).

\subsection{Cell Culture}

P19 cells, belonging to a murine ECC line [34], were used as a source of multipotent murine teratocarcinoma cells, while B16 melanoma cells [35] were used as malignant murine tumor cells followed by porcine embryonic fibroblastic cells, THEPNBS cells [36], engineered to express both tandem dimer Tomato (tdTomato) and enhanced green fluorescent protein (EGFP), and were used as a source of fluorescent normal cells.

P19 and B16 melanoma cells were cultured in Dulbecco's Modified Eagle's Medium (DMEM) and supplemented with glucose at high concentrations (\#11995; Invitrogen Co., Carlsbad, CA, USA), 10\% fetal bovine serum (FBS), as well as 1\% antibiotic-antimycotic solution (\#A5955; Sigma-Aldrich Co., Ltd., St. Louis, MO, USA) whereas THEPNBS cells were cultured in DMEM/Ham's F-12 (\#124; Wako Pure Chemical Industries Ltd., Osaka, Japan), and supplemented with 10\% FBS and 1\% antibiotic- 
antimycotic solution. Since all the cultures were maintained at $37^{\circ} \mathrm{C}$ in an atmosphere of $5 \% \mathrm{CO}_{2}$ in the air, P19, B16 melanoma, and THEPNBS cells were harvested by trypsinization and suspended in Dulbecco's $\mathrm{Ca}^{2+}-\mathrm{Mg}^{2+}$-free phosphate-buffered saline [D-PBS(-)], pH 7.4 (\#14249-95; Nacalai Tesque, Inc., Tokyo, Japan) before the tumor cells inoculation into the adipose tissue. After calculating the number of viable cells using a disposable four-chamber hemocytometer (\#521-10; Funakoshi Co., Ltd., Tokyo, Japan), the cells were diluted to $\sim 5 \times 10^{4}$ cells/ $\mu \mathrm{L}$ in $20 \mu \mathrm{L}$ of D-PBS(-) and $0.1 \%(\mathrm{v} / \mathrm{v}$ ) trypan blue (TB; Trypan Blue Stain 0.4\%; Invitrogen Co.) in a $1.5 \mathrm{~mL}$ tube.

\subsection{IAdICT}

Initiation of cell grafting procedure in the murine adipose tissue was performed using a method described by Sato et al. [32] coupled with some modifications. The schematic representation of the procedure is provided in (Figure 1A). Prior to the procedure, a glass capillary (\#GDC-1; Narishige Scientific Instrument Lab., Tokyo, Japan) was pulled using a micropipette puller (P-97/IVF; Sutter Instrument Company, CA, USA) to form a pointed end while the micropipette tip was broken using micro scissors (\#MB-53; NAPOX, Natsume Co., Ltd., Tokyo, Japan) under a dissecting microscope (SZX10; Olympus, Tokyo, Japan) for changing its inner diameter from 30 to $50 \mu \mathrm{m}$. A solution containing the cells and $0.1 \%(\mathrm{v} / \mathrm{v})$ TB (for visualization of the injected solution) in D-PBS(-) was transferred to the inner surface of a $1.5 \mathrm{~mL}$ tube near the cap using a $200 \mu \mathrm{L}$ tip, following which 1 to $2 \mu \mathrm{L}$ of the solution were sucked using an injection micropipette connected to the mouthpiece (as shown in Figure 1A-a) under observation with a dissecting microscope (Figure 1A-b). Next, a small incision was made on the left dorsal skin of an anesthetized mouse (nude or B6C3F1 mouse), following which, the organs (including ovary, oviduct, and uterus) associated with adipose tissue were pulled and exposed onto the dorsal skin (Figure 1A-c). As the injection micropipette was inserted into the internal part of the adipose tissue under observation using an SZX10 dissecting microscope, the cell-containing solution was injected (Figure 1A-d) while the injection precision was monitored visually based on a rapid color change (from white to blue) at the injection site. Since this procedure was repeated two or three times; hence, three to four injections were administered at each site (Figure 1A-e), and the procedure was repeated for the opposite surface of the adipose tissue, which was exposed by the right dorsal skin incision.

For the juvenile tissue grafting in adipose tissue, juvenile pancreatic tissue was selected as its maturation could be monitored easily via the appearance of insulin-producing cells (IPCs) using Immunohistochemical staining for which the exposed adipose tissue of an adult ( 10 months after birth) B6C3F1 female mouse was partially sliced using micro scissors for creating a small slit (Figure 1B-a) whereas the juvenile pancreas harvested from a 15-day-old B6C3F1 female mouse was sectioned into four parts (right panel of Figure 1B-b) and one tissue piece was inserted into a pancreatic open space using forceps (left panel of Figure 1B-b) followed by the suturing of the edge of the opened space at two sites for the enclosure (arrows in Figure 1B-c). After commencement of surgery, the treated adipose tissue was transferred to its original position, followed by an inspection one month after the surgery. 

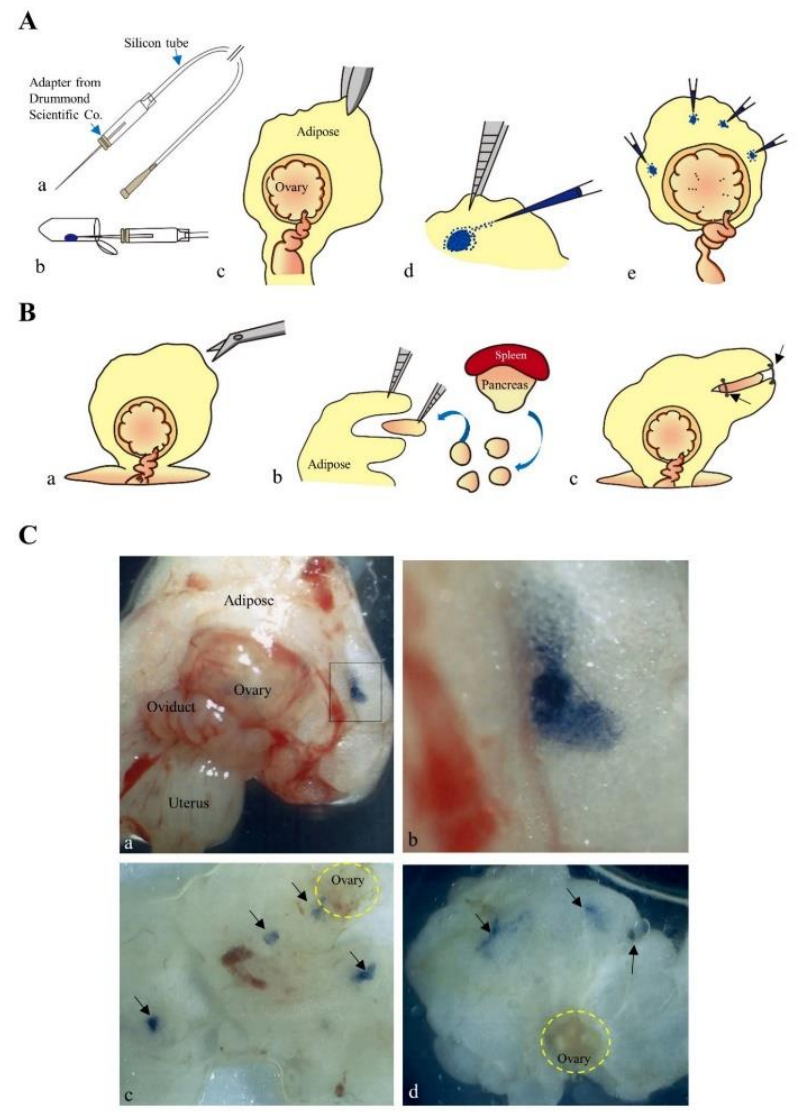

Figure 1 Details of the intra-adipose introduction of cells and tissues (IAdICT) and evidence for solution retention after IAdICT. A. The IAdICT procedure displays the injection of cells into adipose tissue. Cells suspended in a trypan blue (TB)-containing solution were sucked using a glass micropipette attached to a pipette holder (shown in a) under observation with a dissecting microscope (shown in b). In female mice, the reproductive organs (ovary/oviduct/uterus) were exposed under anesthesia, and the exposed adipose tissue was held in position using Aorta-Klemme (\#C-17-40-2; NAPOX, Natsume Co., Ltd) to prevent retraction of the tissue (shown in c). The injection was administered via the micropipette insertion into the adipose tissue held in position using forceps (shown in d), while the whole process required three to four injections as (shown in e). B. The IAdICT procedure, showing the grafting of a small piece of juvenile pancreatic tissue into the adipose tissue, which was first cut using micro scissors, as (shown in a). Juvenile pancreatic tissue from a 15-day-old-mouse was sectioned into four parts using micro scissors (shown in the right panel of b), while one section was inserted into the space created by the adipose tissue incision using forceps (shown in the left panel of $b$ ). The edge of the open adipose tissue was sutured (indicated by arrows in C). C. The fate of TB after IAdICT. When TB was injected into the adipose tissue under observation with a dissecting microscope, the injected dye remained at the site of injection (enclosed by a box in a). Notably, there was no sign of dispersion of TB (shown in b; magnified from the box shown in a). Four injected sites were visible (indicated by blue color) soon after the injection was administered at a site close to the ovary (shown in c). The injected dye was retained close to the ovary even one day after the administration of injections (shown in d). 


\subsection{Histological Analysis}

As the animals were sacrificed the next day after THEPNBS cells grafting, the grafted sections, easily detectable using an SZX10 dissecting microscope due to the presence of TB, were dissected a day after grafting and placed on a glass slide and were squashed gently after placing a cover slip before observing fluorescence, as described below.

Samples were sourced from growing solid tumors and female reproductive tissues surrounded by adipose tissue one and a half months later after grafting, while the sacrificed animals were also subjected to general inspection for tumor invasion in other organs. A part of the dissected tumor tissue was fixed using $4 \%$ paraformaldehyde in D-PBS(-) at $4{ }^{\circ} \mathrm{C}$ for two days, then dehydrated by immersion in $0.25 \%$ sucrose in D-PBS(-) at $4{ }^{\circ} \mathrm{C}$ for two days as well as $0.4 \%$ sucrose (in D-PBS(-)) at $4{ }^{\circ} \mathrm{C}$ for four days followed by embedding of samples in optimal cutting temperature compound (Tissue-Tek ${ }^{\circledR}$ [no. 4583]; Miles Scientific, Naperville, IL, USA) for cryostat sectioning (5 $\mu \mathrm{m}$ in thickness) and staining of few cryostat sections using hematoxylin and eosin stain (H\&E).

Subsequent to the quarter section grafting of the juvenile pancreas, the animals were sacrificed a month later. Thereafter, the grafts were removed, dissected, fixed, and processed for cryostat sectioning using the aforementioned method for solid tumors processing, while some cryostat sections were subjected to immunohistochemical staining, as described below.

\subsection{Immunohistochemical Staining}

After incubation of the cryostat sections in 20\% AquaBlock (\#PP82; East Coast Bio, North Berwick, $\mathrm{ME}$, USA) for $30 \mathrm{~min}$ at $24^{\circ} \mathrm{C}$, they were treated with a goat anti-insulin primary antibody (1:100; \#ab7842; Abcam, Tokyo, Japan) or a mouse anti-glucagon primary antibody (1:100; \#G2654; SigmaAldrich, Tokyo, Japan) for $12 \mathrm{~h}$ at $4{ }^{\circ} \mathrm{C}$ followed by the treatment with fluorescein isothiocyanate (FITC)-conjugated anti-goat IgG (1:250; \#ab6904; Abcam), or Alexa Fluor 594-conjugated anti-mouse IgG (1:250; \#ab150116; Abcam) for one hour at $24{ }^{\circ} \mathrm{C}$. After washing with D-PBS(-) medium, the sections were treated using the mounting medium, and fluorescence was analyzed after treating the sections with 4',6-diamidino-2-phenylindole (DAPI) (\#H-1200; Vector Laboratories, Burlingame, CA, USA).

\subsection{Fluorescence Observation}

Fluorescence in the samples was examined under a fluorescence BX60 microscope using the DM505 (BP460-490 and BA510IF; Olympus) and DM600 (BP545-580 and BA6101F; Olympus) filters, which were used to detect FITC-derived green fluorescence and Alexa Fluor 594-derived red fluorescence, respectively while the DAPI-derived fluorescence was measured using a mirror unit (BP372-456; U-FUW) followed by observation of the $\mathrm{H}$-E-stained sections using a BX60 microscope, recording of the micrographs using a digital camera (FUJIX HC-300/OL; Fuji Film, Tokyo, Japan) attached to the fluorescence microscope, and the printing of images using a Mitsubishi digital color printer (CP700DSA; Mitsubishi, Tokyo, Japan).

All animal experiments were performed in agreement with the guidelines of the Kagoshima University Committee on Recombinant DNA Security and approved by the Animal Care and Experimentation Committee of Kagoshima University (Permit no. 25035 and 25036; dated August 8, 
2013). The surgeries were performed under anesthetization with three agents (medetomidine, midazolam, and butorphanol), and all efforts were made to facilitate a smooth induction.

\section{Results}

\subsection{Successful Entrapment of Transplanted Cells in Adipose Tissues}

The schematic representation of the IAdICT procedure is provided in (Figure 1A) and explained in detail in Section 2.3 of the Materials and Methods. The exposed adipose tissue in the anesthetized B6C3F1 mouse was subjected to IAdICT while being injected a TB solution under a dissecting microscope using a mouthpiece-controlled micropipette. As shown in (Figure 1C-a), the adipose tissue near the ovary was injected once and could be visualized easily based on the color change from white to blue (indicated by the section enclosed by a box in Figure 1C-a). (Figure 1C-b shows the enlarged image of the box shown in Figure 1C-a). Notably, the injected solution did not spread beyond the injection site, suggesting that the dye was confined only to the adipose tissue. The arrows in (Figure 1C-c) indicate that all the four sites were successfully injected while the injected solution was visible even a day after the injection and was retained in the injection site (arrows in Figure 1C-d).

Furthermore, THEPNBS cells [36] were injected into the adipose tissue of adult B6C3F1 mice for confirming the retention of cells introduced via IAdICT in the adipose tissue, followed by an inspection conducted a day after IAdICT, which detected EGFP fluorescence in the injection site without any noticeable cellular breakout (arrows in Figure 2A). 
A

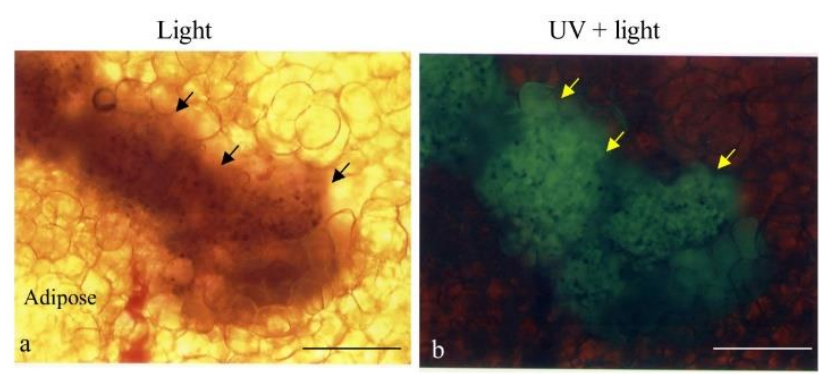

B
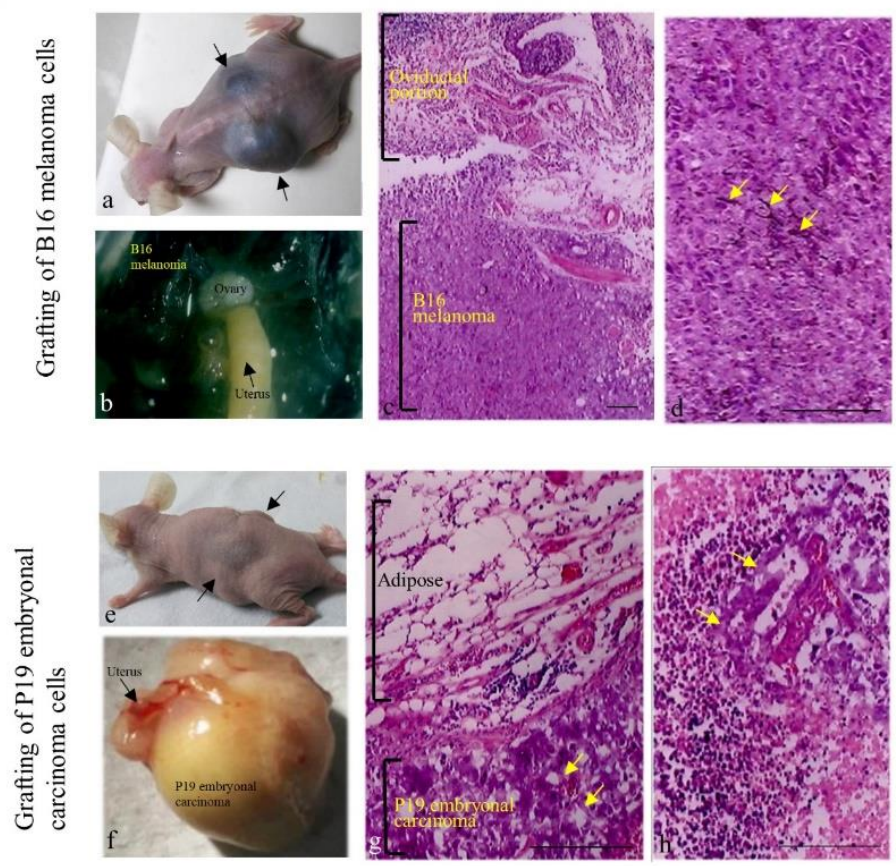

Figure 2 Evidence for cell retention and tumor growth after IAdICT. A. Detection of fluorescent porcine cells (THEPNBS) injected into the adipose tissue. The tissue at the site of the injection (which was easily identifiable based on the presence of trypan blue) was dissected for evaluating EGFP-derived fluorescence using a fluorescence microscope under light (a) or ultraviolet (UV) + light (b) illumination after a day of IAdICT. The arrows indicate the administration of THEPNBS cells into the adipose tissue. Bar: $100 \mu \mathrm{m}$. B. Formation of solid tumors one and a half months after IAdICT. (a-d) Solid tumors were generated after the B16 melanoma cells were grafted in the adipose tissue of nude mice. As solid tumors (arrows in a) were visible one and a half months after IAdICT, their dissection revealed that the ovary and uterus were surrounded by B16 melanoma cells (b). Cryostat tumor sectioning revealed the presence of homogenous cell populations associated with the reproductive organs (c). Some cells were found to exhibit melanin deposition (arrows in d). (e-h) Solid tumors (teratomas) generated after the grafting of P19 embryonal carcinoma cells (ECCS) into the adipose tissue of nude mice that were visible (arrows in e) one and a half months after IAdICT. When these tumors were dissected, the P19 ECCs were found to be associated with the reproductive organs (indicated by arrows in $\mathrm{f}$ ), while cryostat tumor sectioning divulged the presence of differentiated cells (indicated by arrows in g) associated with the adipose tissue and tubular structures in some cases (arrows in h). Bar: $100 \mu \mathrm{m}$. 


\subsection{Formation of Solid Tumors after IAdICT in Mice}

P19 ECCs and B16 melanoma cells were introduced into the nude mice for assessment of the formation of solid tumors by the proliferative tumor cells transplanted in the adipose tissue. When the grafted nude mice were inspected one and a half months after transplantation, solid tumors of $\sim 1.5$ to $2 \mathrm{~cm}^{3}$ had formed in both sides (arrows in Figure 2B-a, e) with $100 \%$ efficiency (2/2 of the grafted adipose for each of the P19 ECCs and B16 melanoma cells). As the reproductive organs (ovary, oviduct, and uterus) were surrounded by the expanding tumors (Figure 2B-b, f), it suggested that these tumors were generated from the adipose layer surrounding the reproductive organs and was also confirmed by cryostat sectioning that the tumors were present in the vicinity of the reproductive organs (Figure $2 \mathrm{~B}-\mathrm{c}$ ) or adipose tissue (Figure 2B-g). The B16 melanoma cell-derived tumors contained homogeneous cell populations with melanin deposits (arrows in Figure 2B-d), while on the contrary, the P19 ECC-derived tumors exhibited differentiation in some cells (arrows in Figure 2B-g, h); however, the degree of multi-differentiation was limited. It was suggested that, unlike original P19 ECCs, the differentiation potential of the P19 ECCs used in the study might have been reduced during cultivation and storage; however, the reason for this remained unclear. There were no appreciable teratomas in the other major organs such as the kidney, heart, intestine, lung, and spleen (data not shown).

\subsection{Maturation of Juvenile Pancreas in the Adipose Tissues after Syngeneic Grafting}

In order to assess whether the juvenile pancreas transplanted into the adipose tissue could proliferate and mature to synthesize functional insulin, a small section of the pancreatic tissue (onefourth section of the 15-day-old pancreas; $3 \sim 4 \mathrm{~mm}^{2}$ in size; the right panel of Figure $3 \mathrm{~A}-\mathrm{a}$ ) was inoculated into a space created by adipose tissue incision, as shown schematically in (Figure 1B-b). The graft growth was easily identified through the dorsal muscle when the skin was peeled off one month after inoculation (arrow in Figure 3A-b). The dissected graft was $\sim 8 \mathrm{~mm}^{2}$ in size and distinguishable from the surrounding adipose tissue since the color of the graft was more yellowish than that of the adipose cells (area enclosed by a circle in Figure 3A-c). Immunohistochemical analysis of the dissected graft indicated that the graft reacted positively to the anti-glucagon and anti-insulin antibodies (Figures 3B-c, d). However, when the cryostat section was inspected in greater detail, the positive reactive areas were segregated from each other as the area positively stained with the anti-glucagon antibodies (considered to contain acinar cells) was not stained by the anti-insulin antibodies (Figure 3B-c, e), whereas the area positively stained with the anti-insulin antibodies (considered to contain islet cells) was not stained by anti-glucagon antibodies (Figure 3B$d, e)$. Therefore, these findings suggested that the graft growing in the adipose tissue could produce both insulin and glucagon. 
A

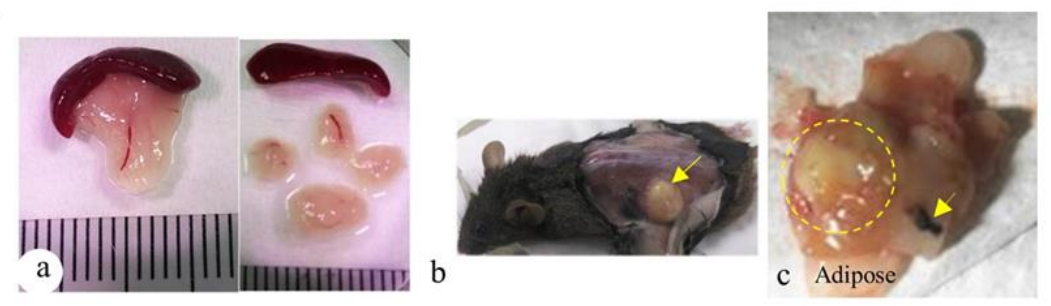

B
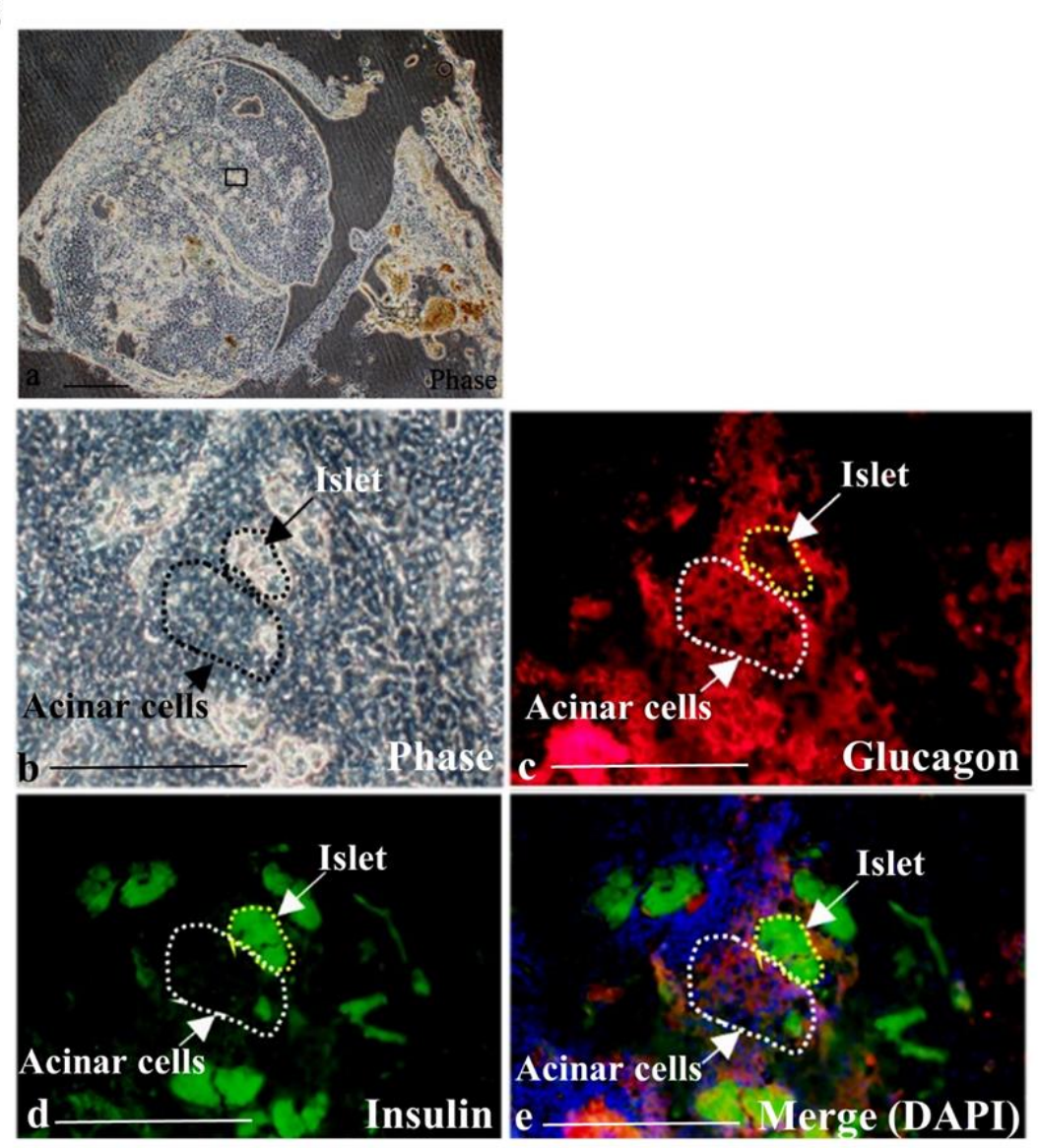

Figure 3 Maturation of juvenile pancreas after IAdICT. A. Grafting of a small piece of the juvenile pancreas into the adipose tissue and isolation of the grafted tissue. The juvenile pancreas (15-day-old) was dissected with the spleen (left panel of a) while the pancreas was divided into four sections using micro scissors (right panel of a). The growing grafts were visible (arrow in b) one month after the insertion of a quarter section of the pancreas into the adipose tissue of a B6C3F1 female mouse. The dissection graft is (shown in c) followed by the growth of the pancreatic tissue (indicated by a dotted circle) in the adipose cell region, with the arrow indicating the suture. B. Immunohistochemical staining of cryostat sections containing the graft shown in (Figure 3A-c). (a) The sections were stained with either anti-glucagon (b) or anti-insulin (c) antibodies. (b-d) Magnified images of the box are shown in (a). The area (indicated by dotted lines; enriched with acinar cells) exhibiting positive staining with anti-glucagon antibodies showed negative staining with anti-insulin antibodies (b vs. c), whereas the area (indicated by the closed lines; enriched with pancreatic $\beta$ cells) exhibiting positive staining with anti-insulin antibodies showed negative staining with anti-glucagon antibodies (c vs. b). Nuclear staining was performed using DAPI (d). Bar: $100 \mu \mathrm{m}$. 


\section{Discussion}

This study aimed to test the appropriateness of adipose tissue for facilitating the growth and differentiation of tumorigenic cells or immature tissues. Although there is limited information on the role of adipose tissues, they primarily store energy in the form of lipids and provide a stable cushioning and insulation to organs. Recently, adipose tissue has been recognized as a major endocrine organ [37], since it produces hormones such as leptin, estrogen, resistin, and cytokines. Furthermore, adipose-derived stem cells, which can be obtained when adipose tissue is present abundantly, are now being considered useful candidates for damaged tissue repair in regenerative medicine $[38,39]$.

It is observed that the retention of the grafted cells at the site of introduction without noticeable cell breakout appears to be a prerequisite factor for confirming the suitability of adipose tissue for the growth and maturation of grafted cells and tissues. Our study confirmed that the injection of a solution containing either only TB or cells (THEPNBS) into the adipose tissue resulted in the retention of the dye or cells at the site of injection without any signs of extensive dispersion when the samples were inspected one day after the injection (see Figures $1 C-d$ and $2 A$ ). This result was contradictory to the findings of a previous study involving the inoculation of cells subcutaneously and beneath the renal capsule, which was frequently associated with the migration of cells from the inoculation site [11]. Our previous study conducted on inoculation of tumorigenic cells (including human iPSCs and F9 murine teratocarcinoma cells) into the pancreatic parenchyma observed that the grafted cells exhibited better proliferation activity as well as successfully formed solid tumors with $100 \%$ efficiency [32]. In our study, it was reported that the cells were retained at the injection sites when an inspection was performed one day after inoculation, while even a small number $\left(\sim 10^{3}\right)$ of cells could survive and proliferate in the in vivo environment [32]. Histological analysis of the tumors derived from human iPSCs revealed the presence of various types of differentiated cells, suggesting that the pancreatic environment does not affect the ability of iPSCs to differentiate into various cell types. Hence, it can be corroborated that adipose tissue could be considered as an ideal material for promoting the survival, proliferation, and differentiation of multipotent tumorigenic cells due to the exhibition of cellular differentiation by solid tumors derived from P19 ECCs, although their differentiation potential appears to be limited (see Figure $2 \mathrm{~B}-\mathrm{g}, \mathrm{h}$ ). In this context, further follow-up studies using iPSCS or ESCS, which are more vulnerable to multi-lineage differentiation, could be deemed necessary for confirming the suitability of adipose tissue for the differentiation of multipotent tumorigenic cells.

Owing to the fact that adipose tissue can promptly support the proliferation and differentiation of multipotent tumorigenic cells led our study to investigate whether a small section of juvenile pancreatic tissue inoculated into the murine adipose can grow and mature. As depicted in (see Figure 1B), a fragment of the pancreatic tissue was inserted into a space formed after incision using microscissors. The resulting grafts were quite large $\left(3 \sim 4 \mathrm{~mm}^{2} v s . \sim 8 \mathrm{~mm}^{2}\right.$; see right panel of Figure $3 A-a$ vs. Figure $3 A-b, c)$ when inspected one month after grafting, and had well-organized structures that were responsive to the antibodies specific for glucagon or insulin (see Figure $3 \mathrm{~B}$ ), suggesting the unerring efficacy of this approach for inducing the juvenile pancreatic tissues maturation. Hence, this concept could be put forth that as attempts were contrived for inducing the in vivo maturation of pancreatic $\beta$ cells by transplanting intermediate cells (which are produced from human iPSCs in vitro and express the $\beta$ cell-specific transcript of pancreatic and duodenal homeobox factor- 1 ) in the 
nude mice pancreatic parenchyma, it was observed that IPCs were generated in the grafts, which were later found to be strongly stained by anti-insulin antibodies when an inspection was undertaken six months after grafting [39, 40]. Analogous to these findings, IPCs may also be generated when human iPSC-derived intermediate cells are implanted into the adipose tissue of nude mice via the IAdICT procedure.

\section{Conclusion}

To conclude, this is the first study that depicts the feasibility of using adipose tissue as a tissue conducive to the proliferation and differentiation of multipotent tumorigenic cells as solid tumors. Our study's novel approach, named "IAdICT", appears to be an accurate and precise alternative for the "in vivo teratoma formation assay", as it provides solid tumor formation with a $100 \%$ success rate using a small number $\left(<10^{3}\right)$ of tumor cells. However, since IAdICT is an invasive procedure, it requires surgical interventions performed by duly experienced personnel with fully supporting anesthetic assistance for the experimental subjects to streamline the process. On the contrary, subcutaneous implantation can also be done via injections that permit forthright monitoring of teratoma development [16]. Furthermore, adipose tissue was discovered as a suitable medium for the maturation of juvenile tissues, including juvenile pancreatic tissue, after a procedural inoculation of a small section of the tissue in the adipose tissue layers. Hence, this approach may be of paramount importance in additional follow-up studies, which might be instrumental in extracting the usage of adipose tissue judiciously, as well as could further pinpoint and emphasize the formation of matured 3-D functional tissues.

\section{Acknowledgments}

This study was partly supported by a grant (no. 19 K06372 for M.S.; no. 18 K09839 for E.I.) from The Ministry of Education, Science, Sports, and Culture, Japan.

\section{Author Contributions}

M.S. conceived and designed the study, drafted the manuscript, performed the experiments, and revised the manuscript. Y.K., E.I., E.A., S.W. and I.S. critically revised the manuscript.

\section{Funding}

This article received no external funding.

\section{Competing Interests}

The authors have declared that no competing interests exist.

\section{References}

1. Solter D. From teratocarcinomas to embryonic stem cells and beyond: A history of embryonic stem cell research. Nat Rev Genet. 2006; 7: 319-327.

2. Damjanov I, Andrews PW. The terminology of teratocarcinomas and teratomas. Nat Biotechnol. 2007; 25: 1212. 
3. De Coppi P, Bartsch G Jr, Siddiqui MM, Xu T, Cesar CS, Laura P, et al. Isolation of amniotic stem cell lines with potential for therapy. Nat Biotechnol. 2007; 25: 100-106.

4. Takahashi K, Tanabe K, Ohnuki M, Narita M, Ichisaka T, Tomoda K, et al. Induction of pluripotent stem cells from adult human fibroblasts by defined factors. Cell 2007; 131: 861-872.

5. Yu J, Vodyanik MA, Smuga-Otto K, Antosiewicz-Bourget J, Frane L, Tian S, et al. Induced pluripotent stem cell lines derived from human somatic cells. Science 2007; 318: 1917-1920.

6. Brivanlou $A H$, Gage $F H$, Jaenisch R, Jessell $T$, Douglas $M$, Janet R. Setting standards for human embryonic stem cells. Science 2003; 300: 913-916.

7. Gertow K, Przyborski S, Loring JF, Auerbach JM, Epifano O, Otonkoski T, et al. Isolation of human embryonic stem cell-derived teratomas for the assessment of pluripotency. Curr Protoc Stem Cell Biol. 2007 Chapter 1:Unit1B.4. doi: 10.1002/9780470151808.sc01b04s3.

8. Adewumi O, Aflatoonian B, Ahrlund-Richter L, Amit M, Andrews PW, Beighton G, et al. Characterization of human embryonic stem cell lines by the International Stem Cell Initiative. Nat Biotechnol. 2007; 25: 803-816.

9. Muller FJ, Goldmann J, Loser P, Loring JF. A call to standardize teratoma assays used to define human pluripotent cell lines. Cell Stem Cell 2010; 6: 412-414.

10. Cooke MJ, Stojkovic M, Przyborski SA. Growth of teratomas derived from human pluripotent stem cells is influenced by the graft site. Stem Cells Dev. 2006; 15: 254-259.

11. Prokhorova TA, Harkness LM, Frandsen U, Ditzel N, Schrøder HD, Burns JS, et al. Teratoma formation by human embryonic stem cells is site dependent and enhanced by the presence of Matrigel. Stem Cells Dev. 2009; 18: 47-54.

12. Blum B, Benvenisty N. Clonal analysis of human embryonic stem cell differentiation into teratomas. Stem Cells 2007; 25: 1924-1930.

13. Gutierrez-Aranda I, Ramos-Mejia V, Bueno C, et al. Human induced pluripotent stem cells develop teratoma more efficiently and faster than human embryonic stem cells regardless the site of injection. Stem Cells 2010; 28: 1568-1570.

14. Honda A, Hirose M, Hatori M, Matoba S, Miyoshi H, Inoue K, et al. Generation of induced pluripotent stem cells in rabbits: Potential experimental models for human regenerative medicine. J Biol Chem. 2010; 285: 31362-31369.

15. Ritner C, Bernstein HS. Fate mapping of human embryonic stem cells by teratoma formation. J Vis Exp. 2010; 42: 2036.

16. Gropp M, Shilo V, Vainer G, Miri Gov M, Gil Y, Khaner H, et al. Standardization of the teratoma assay for analysis of pluripotency of human ES cells and biosafety of their differentiated progeny. PLoS ONE 2012; 7: e45532.

17. Liu Z, Tang Y, Lu S, Zhou J, Du ZY, Duan CM, et al. The tumourigenicity of iPS cells and their differentiated derivates. J Cell Mol. Med. 2013; 17: 782-791.

18. Gertow K, Wolbank S, Rozell B, Sugars R, Andäng M, Parish CL, et al. Organized development from human embryonic stem cells after injection into immunodeficient mice. Stem Cells Dev. 2004; 13: 421-435.

19. Przyborski SA. Differentiation of human embryonic stem cells after transplantation in immunedeficient mice. Stem Cells 2005; 23: 1242-1250.

20. Stojkovic P, Lako M, Stewart R, Przyborski S, Armstrong L, Evans J, et al. An autogeneic feeder cell system that efficiently supports growth of undifferentiated human embryonic stem cells. Stem Cells. 2005; 23: 306-314. 
21. Werbowetski-Ogilvie TE, Bosse M, Stewart M, Schnerch A, Ramos-Mejia V, Rouleau A, et al. Characterization of human embryonic stem cells with features of neoplastic progression. Nat Biotechnol. 2009; 27: 91-97.

22. Peterson SE, Tran HT, Garitaonandia I, Han S, Nickey SK, Leonardo T, et al. Teratoma generation in the testis capsule. J Vis Exp. 2011; 57: e3177.

23. Lee AS, Tang C, Cao F, Xie X, et al. Effects of cell number on teratoma formation by human embryonic stem cells. Cell Cycle. 2009; 8: 2608-2612.

24. Amit M, Margulets V, Segev H, Shariki K, Laevsky I, Coleman R, et al. Human feeder layers for human embryonic stem cells. Biol Reprod. 2003; 68: 2150-2156.

25. Choo AB, Padmanabhan J, Chin AC, Oh SK. Expansion of pluripotent human embryonic stem cells on human feeders. Biotechnol Bioeng. 2004; 88: 321-331.

26. Plaia TW, Josephson R, Liu Y, Zeng X, Ording C, Toumadje A, et al. Characterization of a new $\mathrm{NIH}$-registered variant human embryonic stem cell line, BG01V: A tool for human embryonic stem cell research. Stem Cells. 2006; 24: 531-546.

27. Tzukerman M, Rosenberg T, Reiter I, Ben-Eliezer S, Denkberg G, Coleman R, et al. The influence of a human embryonic stem cell-derived microenvironment on targeting of human solid tumor xenografts. Cancer Res. 2006; 66: 3792-3801.

28. Hentze H, Soong PL, Wang ST, Phillips BW, Putti TC, Dunn NR. Teratoma formation by human embryonic stem cells: Evaluation of essential parameters for future safety studies. Stem Cell Res. 2009; 2: 198-210.

29. Nishimura K, Nakagawa T, Sakamoto T, Ito J. Fates of murine pluripotent stem cell-derived neural progenitors following transplantation into mouse cochleae. Cell Transplant. 2012; 21: 763-771.

30. Ono $H$, Obana A, Usami $Y$, Sakai $M$, Nohara K, Egusa $H$, et al. Regenerating salivary glands in the microenvironment of induced pluripotent stem cells. BioMed Res Int. 2015; 293570: 11.

31. Gordeev OF, Nikonova TM. Development of experimental tumors formed by mouse and human embryonic stem and teratocarcinoma cells after subcutaneous and intraperitoneal transplantations into immunodeficient and immunocompetent mice. Cell Transplant. 2013; 22: 1901-1914.

32. Sato M, Saitoh I, Murakami T, Kubota N, Nakamura S, Watanabe S, et al. Intrapancreatic parenchymal injection of cells as a useful tool for allowing a small number of proliferative cells to grow in vivo. Int J Mol Sci. 2017; 18: 1678.

33. Birbrair A, Zhang T, Wang ZM, Messi ML, Enikolopov GN, Mintz A, et al. Role of pericytes in skeletal muscle regeneration and fat accumulation. Stem Cells Dev. 2013; 22: 2298-2314.

34. McBurney MW, Rogers BJ. Isolation of male embryonal carcinoma cells and their chromosome replication patterns. Dev Biol. 1982; 89: 503-508.

35. Hu F, Lesney PF. The isolation and cytology of two pigment cell strains from B16 mouse melanomas. Cancer Res. 1964; 24: 1634-1643.

36. Sato $M$, Ohtsuka M, Miura H, Miyoshi K, Watanabe S. Determination of the optimal concentration of several selective drugs useful for generating multi-transgenic porcine embryonic fibroblasts. Reprod Dom Anim. 2012; 47: 759-765.

37. Kershaw EE, Flier JS. Adipose tissue as an endocrine organ. J Clin Endocrinol Metab. 2004; 89: 2548-2556. 
38. Tsuji W, Rubin JP, Marra KG. Adipose-derived stem cells: Implications in tissue regeneration. World J Stem Cells. 2014; 6: 312-321.

39. De Francesco F, Matta C, Riccio M, Sbarbati A, Mobasheri A. Reevolution of tissue regeneration: From recent advances in adipose stem cells to novel therapeutic approaches. Stem Cells Int. $2021 ; 2179429$.

40. Kiyokawa $Y$, Sato $M$, Noguchi $H$, Inada E, Iwase $Y$, Kubota $N$, et al. Drug-induced naïve iPS cells exhibit better performance than primed iPS cells with respect to the ability to differentiate into pancreatic $\beta$-cell lineage. J Clin Med. 2020; 9: E2838.

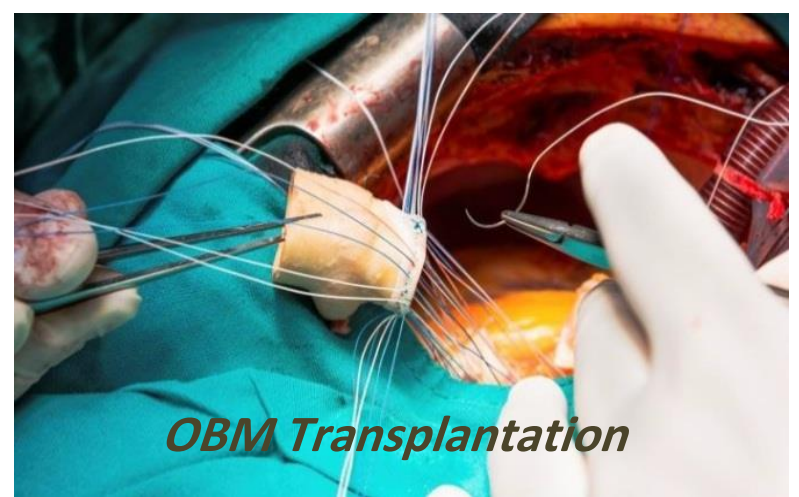

Enjoy OBM Transplantation by:

1. Submitting a manuscript

2. Joining in volunteer reviewer bank

3. Joining Editorial Board

4. Guest editing a special issue

For more details, please visit: http://www.lidsen.com/journals/transplantation 